\title{
Destinos Turísticos Inteligentes, un nuevo aporte conceptual para el desarrollo local en Ecuador. Caso "Portoviejo Ciudad Creativa UNESCO"
}

\author{
Smart Tourist Destinations, a new conceptual contribution for local development in Ecuador. Case \\ of "Portoviejo Creative City UNESCO"
}

Destinos turísticos inteligentes, uma nova contribuição conceitual para o desenvolvimento local no Equador. Case "Portoviejo Cidade Criativa UNESCO"

\author{
Ángel Guillermo Félix Mendoza \\ ORCID: https://orcid.org/0000-0003-1586-1068 \\ Escuela Superior Politécnica Agropecuaria de Manabí Manuel Felix López, Ecuador \\ E-mail: guillofelix@gmail.com \\ Johnny Patricio Bayas Escudero \\ ORCID: https://orcid.org/0000-0002-3413-4352 \\ Escuela Superior Politécnica Agropecuaria de Manabí Manuel Felix López, Ecuador \\ E-mail: johnnybayas@gmail.com \\ José Rafael Vera Vera \\ ORCID: https://orcid.org/0000-0003-1721-8770 \\ Escuela Superior Politécnica Agropecuaria de Manabí Manuel Felix López, Ecuador \\ E-mail: jrafaw.4@gmail.com \\ Washington Fernando Veloz Camejo \\ ORCID: https://orcid.org/0000-0002-7113-2049 \\ Escuela Superior Politécnica Agropecuaria de Manabí Manuel Felix López, Ecuador \\ E-mail: fernandovelozc@gmail.com \\ Joffre Ramón Moreira Pico \\ ORCID: https://orcid.org/0000-0001-6961-3188 \\ Escuela Superior Politécnica Agropecuaria de Manabí Manuel Felix López, Ecuador \\ E-mail: joramopi@hotmail.com
}

\begin{abstract}
Resumen
La actividad turística cada día se vuelve más competitiva, el crecimiento exponencial de la tecnología hace posible el mejoramiento de las comunicaciones entre negocios y clientes finales, la aparición de nuevas aplicaciones facilitan al turista la adquisición de servicios de manera más fácil. Por esto y muchas cosas más, la administración pública en países turísticamente desarrollados como España apuesta a la certificación de Destinos Turísticos Inteligentes (DTI) con criterios relacionados a la accesibilidad, sostenibilidad, gobernanza, tecnología e innovación. La presente revisión de literatura utiliza herramientas básicas de análisis de contenido, para clasificar la información de documentos, manuales y artículos especializados en diversas bases de datos, los mismos que buscan sentar las bases que sirvan como referencia para nuevos estudios turísticos enfocados en este ámbito, novedoso para Ecuador, pero no tanto alrededor del mundo. Portoviejo es una ciudad recientemente declarada Ciudad Creativa por la UNESCO, la misma busca promover un nuevo modelo de gestión turístico, que haga uso de su patrimonio cultural, encabezado por la diversidad gastronómica, y a su vez poder potenciar este desarrollo con la evolución hacia un destino inteligente. Palabras clave: Gestión de destinos; Gastronomía; Ciudades creativas; Destinos inteligentes; Portoviejo.
\end{abstract}

\begin{abstract}
Tourism activity becomes more competitive every day, the exponential growth of technology makes it possible to improve communications between businesses and final customers, the emergence of new applications makes it easier for tourists to acquire services. For this and many other things, the public administration in tourist developed countries such as Spain is committed to the certification of Smart Tourist Destinations (STD) with criteria related to accessibility, sustainability, governance, technology and innovation. This literature review uses basic content analysis tools to classify the information from documents, manuals and specialized articles in various databases, which seeks to raise the foundations that serve as a reference for new tourism studies focused on this field. novel for Ecuador, but not so much around the world. Portoviejo is a city recently declared as a Creative City by UNESCO, and it seeks to promote a new model of tourism management, which makes use of its cultural heritage, led by gastronomic diversity, and in turn be able to promote this development with the evolution towards a smart destination. Keywords: Destination management; Gastronomy; Creative cities; Smart destinations; Portoviejo.
\end{abstract}




\section{Resumo}

A atividade turística torna-se cada vez mais competitiva, o crescimento exponencial da tecnologia permite melhorar a comunicação entre empresas e clientes finais, o aparecimento de novas aplicações facilita a aquisição de serviços pelos turistas. Por isso e por muitas outras coisas, a administração pública em países turísticos desenvolvidos como a Espanha está comprometida com a certificação de Smart Tourist Destinations (DTI) com critérios relacionados à acessibilidade, sustentabilidade, governança, tecnologia e inovação. Esta revisão bibliográfica utiliza ferramentas básicas de análise de conteúdo para classificar as informações de documentos, manuais e artigos especializados em diversas bases de dados, que buscam lançar as bases que servem de referência para novos estudos de turismo nesta área. Romance para o Equador, mas não é assim muito em todo o mundo. Portoviejo é uma cidade recentemente declarada Cidade Criativa pela UNESCO, pretende promover um novo modelo de gestão do turismo, que valorize o seu património cultural, pautado pela diversidade gastronómica, e por sua vez seja capaz de promover este desenvolvimento com a evolução para um destino inteligente.

Palavras-chave: Gestão de destinos; Gastronomia; Cidades criativas; Destinos inteligentes; Portoviejo.

\section{Introducción}

El desarrollo acelerado de la tecnología ha generado cambios drásticos en todos los sectores de la sociedad, el consumismo y la demanda emergente de movilidad, han dado paso a distintas propuestas como la de las Smart Cities [ciudades inteligentes] (Bernal citado por Mendoza, 2016) que según Buhalis y Amaranggana (2014) han dado origen al concepto de Smart Tourism Destination [Destinos Turísticos Inteligentes], donde las tecnologías de la información y la comunicación (TIC) desempeñan un rol muy importante para la creación y fortalecimiento de la competitividad (Berne, Garcia-Gonzalez, y Mugica, 2012; Navío-Marco, Ruiz-Gómez, y Sevilla-Sevilla, 2018); y garantizan un desarrollo sostenible, eficacia de los recursos disponibles además de mejorar la calidad de vida de su población.

El turismo es uno de los sectores con mayor direccionamiento a la digitalización; tecnologías como big data, redes sociales, chatbots, nanotecnología, realidad virtual, el IoT (Internet de las cosas) están impactando fuertemente en los hábitos de los consumidores y las experiencias generadas (Fernández et al. 2017); el volumen de reservas a través de la web mediante booking.com y Expedia, acapararon el 70\% sobre las Operadoras turísticas (HOSTELTOUR, 2019); lo que evidencia el impacto que tiene en el sector; esta digitalización que ha permitido que los viajeros tengan más control sobre la selección de sus viajes; con mayor poder de negociación, es más selectivo y exigente, ha dejado de lado los paquetes organizados y ha priorizado los programas personalizados en los que busca vivir experiencias; de hecho, el sector turístico se encuentra entre los cinco de mayor madurez digital, por delante de las finanzas y el comercio (Huete, 2019. p. 1).

Este fenómeno de transformación digital, ha derivado en el desarrollo de dispositivos electrónicos cada vez más sofisticados, que en aspecto tecnológico buscan mejorar la experiencia del usuario y en el contexto turístico permiten brindar una mejor experiencia al turista durante su visita. En este sentido, la tecnología móvil ha tomado especial connotación en los últimos años y se constituido como un importante canal de contratación de productos y servicios turísticos (Wei-Han Tan y Keng-Boon, 2018). Por esta razón, la dependencia de un destino turístico inteligente (DTI) a los avances de la tecnología móvil es cada vez más fuerte, y la necesidad de conocer su aplicabilidad, más imperativa. Kim y Kim (2017) destacan tres expectativas relevantes de la tecnología móvil: la creación de experiencias innovadoras para los consumidores, el fomento de la ventaja competitiva sostenible para los destinos turísticos y los proveedores relacionados a este sector, y la creación de competencias de sostenibilidad para el turismo inteligente.

A partir la cuarentena generada por la pandemia del COVID 19, la actividad turística en todo el mundo se detuvo, según la OMT en el año 2018 se movilizaron 1400 millones de turistas, que representaron un incremento de 5\% en relación al año anterior, y fue el noveno año de crecimiento sostenido con ingresos cercanos a 1,7 billones de dólares, siendo uno de los principales generadores de crecimiento económico y desarrollo (Organización Mundial del Turismo OMT, 2019), y de acuerdo a los primeros datos la reducción en el primer trimestre 2020 ya es del 57\% que representan aproximadamente 80.000 millones de dólares de pérdidas para el sector, afectando no solo a la supervivencia de personas, sino también a la sociedad en todas sus 
dimensiones (Navarro Jurado y Ortega 2020).

En estas circunstancias la tecnología se ha convertido en un aliado que ofrece un sinnúmero de opciones para adaptarse al distanciamiento físico, producto de la pandemia; así encontramos que las redes sociales han jugado un papel preponderante durante la pandemia, en España se utilizó para detectar el número de infectados en base a un Web-Service que permite compartir los datos de cualquier territorio para ser analizados a tiempo real (Linares et al. 2020. P.2). De hecho, la innovación tecnológica en el turismo, tiene como uno de los principales referentes a España, donde a partir del año 2002 se crea la Sociedad Mercantil Estatal para la Gestión de la Innovación y las Tecnologías Turísticas, S.A. (SEGITTUR), organismo dependiente del Ministerio de la Secretaría de estado de Turismo, responsable de impulsar la innovación (I+D+i) en el sector turístico español, tanto en el sector público (nuevos modelos y canales de promoción, gestión y creación de destinos inteligentes, etc.) como en el sector privado (apoyo a emprendedores, nuevos modelos de gestión sostenible y más competitivo, exportación de tecnología española) (SEGITTUR, 2020), tiene 4 ejes de acción definidos, la innovación, internacionalización, emprendedores y promoción.

A pesar del fuerte impacto que ha tenido la pandemia en España, su sector turístico es uno de los primeros en reactivarse con una demanda futura muy prometedora, encabeza el ranking mundial de reservas turísticas realizadas en los últimos siete días para los próximos meses con un 31,83\% del total a día 9 de junio (La Sexta, 2020). Todo esto producto de un proceso de uso adecuado de la innovación y la tecnología, que les ha permitido mitigar el impacto socioeconómico y acelerar su proceso de recuperación; según Enrique Martínez presidente de SEGITTUR, La tecnología contribuye a mejorar la toma decisiones, priorizar medidas y anticiparse. Todo ello con el objetivo de lograr un turismo más inteligente y sostenible (SEGITTUR 2020a).

Según Ángeles Rubio experta de Hosteltur, España deberá redireccionar los flujos de los turistas, mediante el uso de herramientas como Smart Tourism, el E-commerce y Big Data, lo cual le permitirá mejorar la integración de las plataformas de reservas; a esto se deberá sumar la aplicación de estrictos protocolos sanitarios, que permitan asegurar procesos de seguridad integral para todos sus pasajeros; todo esto evidencia que los 18 años de apostarle a la innovación tecnológica, le permite a España, recuperar en el mediano plazo, un gran porcentaje de su demanda turística internacional.

De acuerdo a las proyecciones de la OMT entre julio y septiembre del presente año, ya comenzará a reactivarse la demanda interna, mientras que en el cuarto trimestre y principalmente en 2021 comenzaría a reactivarse la demanda internacional (OMT, 2020), siendo la innovación y digitalización del sector, la principal oportunidad, ya que la pandemia aceleró la generación y uso de herramientas tecnológicas como Zoom, Meet y las apps; se ha incrementado el uso del celular ya que se ha hecho evidente el potencial de las redes sociales como instrumento de inclusión del consumidor en el desarrollo de nuevas experiencias y productos (Iglesias et al. 2017).

Otra oportunidad es la renovación de los modelos de negocios sostenibles, dejando de lado aquellos que generan la turismofobia producto de la masificación de los destinos y la turistificación, término que vincula al turismo como uno de los grandes ejes de la globalización y el consiguiente movimiento amplio de personas, bienes y capitales que ha generado la destrucción de varios recursos y atractivos turísticos en el mundo.

También una prioridad es la sostenibilidad y reorientación de las actividades hacia los segmentos que se generarán con el turismo de proximidad, entendido como la antítesis del turismo masivo, siendo el desarrollo de actividades turísticas dentro de los límites geográficos entre los cuales un individuo se desplaza en su rutina regular de vida, donde se prioriza la vivencia o experiencia turística (Soria \& Llurdés Coit, 2013). Esta variante implica la aplicación de nuevas normas sociales y un mayor énfasis al desarrollo endógeno, siendo la población local agente activo de su propio desarrollo (Soria y Llurdés Coit, 2013)

También se debe aprovechar la desescalada basada en la "nueva normalidad" la cual está llevando a la generación de 
una diversidad de protocolos que buscan reducir los temores a viajar, adecuándose a los requerimientos y necesidades de los clientes, cuya mayor cautela a la hora de decidir cuánto, dónde y cómo viajar puede, igualmente, ser pasajera (coyuntural) o instalarse con vocación de permanencia (estructural) (Vargas 2020); siendo importante recordar que el sector turístico en las últimas décadas ha afrontado numerosas crisis epidemiológicas, el MERS (2012-2015), el H1N1 (2009) o el SARS (2003), siendo este último el que mayor incidencia ha tenido sobre el turismo internacional, antes de COVID 19 (Menchero Sánchez 2020. p. 107); pero cada uno de estos han impulsado el incremento de medidas coordinadas entre la OMT y la Organización Mundial de la Salud (OMS), a través de la toma de decisiones, y medidas de control.

La adaptación de las empresas turísticas y los destinos a los nuevos paradigmas sociales y de negocios que se han generado producto de la pandemia, donde la tecnificación de la sociedad a través de las TIC, motiva cambios sustanciales en la estructura funcional del sistema turístico, partiendo desde las políticas de gestión pública y su incidencia sobre todo cada uno de los elementos de los destinos turísticos (Palomeque 2016).

En el caso del Ecuador, los efectos causados por el COVID 19, han sido considerables, la caída de ingresos por turismo bordea el 70\%, lo que ha generado una escalada masiva de despidos en diferentes empresas turísticas, como hoteles, restaurantes, agencias de viajes, (El Universo, 2020), todo esto producto de las cancelaciones de reservas de cientos de turistas nacionales y extranjeros, siendo imposible ocultar la realidad: todos los negocios (de todos los tamaños) en todos los sectores de la economía están sintiendo el impacto del COVID-19 (Ortiz y Noboa, 2020. p. 2), que también ha incidido en las estructuras sociales, familiares y laborales generando una dualidad de efectos positivos y negativos, producto del confinamiento obligado, que ha forzado a muchos a cerrar negocios, quedarse en la desocupación o aprovechar para desarrollar emprendimientos por oportunidad, generando nuevos esquemas de trabajo utilizando el internet o las plataformas de comunicación (Santillan, 2020. p.1).

La reactivación es un proceso que lentamente ha iniciado en el Ecuador, desde el 17 de marzo, el gobierno dispuso que cafeterías y restaurantes, operen solo a través de servicio a domicilio, incrementándose el uso del servicio Delivery, a través de: Ubereats, DeliveryEc, Glovo, Rappi, Tipti, Mercadito, etc.; Frente a la emergencia los pedidos han aumentado en $1300 \%$ en las tres ciudades en donde Tipti está presente (Quito, Guayaquil y Cuenca) (El Comercio 2020a), en varios de estos se aplica normas de asepsia, como el uso de mascarilla, guante, gel.

Tomando las premisas de la OMT, sobre la reactivación del sector turístico, el Ministerio de Turismo del Ecuador (MINTUR) ha puesto como una de sus prioridades la recuperación del turismo interno, partiendo desde los destinos rurales cercanos a las ciudades (MINTUR, 2020a), para posteriormente habilitar productos combinados con Perú y Colombia. De igual manera a partir del 22 de mayo, presentó los protocolos de bioseguridad para la reapertura de los establecimientos de alojamiento turístico en estos se señalan los protocolos de arribo de los clientes, las medidas para alojamiento, limpieza y desinfección, así como las medidas de protección que deberán cumplir el personal.

Lamentablemente en el Ecuador, la innovación tecnológica no ha logrado despegar, a pesar de tener un Sistema Nacional de Ciencia, Tecnología e Innovación, todavía no ha dado el "salto", según los expertos, hacia la tercera fase del desarrollo científico-tecnológico (La Hora 2019).

El nuevo entorno socio-económico producido por el COVID 19, encontró un sector público turístico con graves problemas de financiamiento, para la promoción y difusión internacional; y un sector privado, con problemas de flujo de caja, financiamiento, planificación; estas limitaciones ha generado que el país tenga un descenso dramático en el ranking de competitividad turística mundial, pasando del puesto 57 al 70 (World Economic Forum, 2019).

Portoviejo, capital de la provincia de Manabí, es la sexta ciudad más poblada del Ecuador, luego del terremoto de abril del 2016, la ciudad empezó un proceso de reconstrucción, en diferentes áreas y sectores de la ciudad. A partir del 30 de octubre del 2019, fue declarada como ciudad creativa por parte de La Organización de las Naciones Unidas para la Educación, 
la Ciencia y la Cultura (UNESCO) en el ámbito de la gastronomía, como reconocimiento a la diversidad culinaria tradicional, apuntalada por la declaratoria de la cocina tradicional manabita como patrimonio cultural inmaterial del Ecuador.

El Gobierno Autónomo Descentralizado (GAD) cantonal, ha hecho énfasis dentro de su planificación a la vialidad, apoyo productivo, electricidad y seguridad, como prioridades post terremoto, ya que pesar del lanzamiento de la Agenda Portoviejo ciudad digital 2015-2019, en la cual se buscaba reducir la exclusión y la brecha digital, donde se priorizó las dimensiones inteligentes en el denominado modelo de territorio digital priorizando la infraestructura, el gobierno en línea con ejes temáticos y productivos (GAD de Portoviejo 2019), no ha sido posible su implementación.

Las nuevas tendencias sustentadas en la tecnología facilitan la gestión de la actividad turística, que permiten que el sector público, conozca tendencias, necesidades y expectativas de los turistas y aplicar de manera adecuada la inteligencia de mercados, facilitando la gestión, integración, el control y la toma de decisiones; pero, las limitantes que tiene Portoviejo, parten de la pobre infraestructura tecnológica y la limitada aplicación de las tecnologías de la información en el sector turístico en su gestión.

Con la innovación se ha generado nuevos modelos de gestión de los negocios turísticos en base a entornos 2.0 y ligados a la profesionalización de herramientas de distribución, sustentando en el denominado turismo colaborativo (economía de intercambio), ejemplos de esto son Airbnb, Kayak, entre otros; además, los nuevos segmentos turísticos se caracterizan por demandar productos y servicios flexibles y más personalizados, orientados a mejorar su competitividad; pero el sector turístico de Portoviejo, mantiene una infraestructura, que, a pesar de ser renovada luego del terremoto de 2016, su gestión no se adapta a las necesidades de esa nueva demanda; Portoviejo al igual que muchas ciudades del país, promueve servicios que en mucho de los casos son aislados, son muy pocos los productos turísticos que se han gestado basados en las necesidades de la demanda real y potencial, esto debido a la ausencia de data, que facilite la comprensión y generación de nuevas ofertas.

Además, las nuevas tendencias del marketing turístico digital, basado en la creación de nuevas experiencias (turismo experiencial), basado en las necesidades del turista (marketing emocional) para conectarse con sus clientes, donde se maximiza la automatización, dejando de lado los (Customer relationship management) CRM [Administración basada en la relación con los clientes] que permitía gestionar lo comercial, marketing y posventa, por herramientas sustentadas en la inteligencia artificial; que son capaces de gestionar una mayor cantidad de datos de los clientes, facilitando una mayor y mejor segmentación y fidelización de los clientes; apoyado en las redes sociales, que permiten una comunicación más directa y cercana con los clientes, a través de las cuales se puede comunicar promociones, descuentos.

\section{Metodología}

El presente trabajo de revisión es de naturaleza cualitativa debido a que dicho método permite dar una interpretación del caso de estudio por parte del investigador con sus opiniones basadas en la evidencia obtenida en este caso, del extenso material bibliográfico consultado (Soares, Moreira, Pereira, Shitsuka, 2018). Así mismo, se toma como base el tipo de investigación exploratoria a partir del conocimiento del objeto de estudio, debido al acercamiento que se hace a una temática nueva para el área de las ciencias que rodean al turismo y de alta incidencia a nivel mundial. Los métodos utilizados están relacionados al análisis y síntesis de la información procesada (analítico-sintético). Se realizó una revisión de literatura especializada, de estudios de casos basados en metodologías, entornos y enfoques analíticos no convencionales de investigación (Buchanan \& Bryman, 2011).

Las actividades se han centrado en primer lugar, a realizar una revisión y síntesis sistemática de la literatura existente, con esto se ha hecho una aproximación al estado del arte, relacionado a la temática central, en este caso los DTI y sus componentes conceptuales. Para esta labor se ha tomado como referencia en primera instancia diversos estudios que contemplen las relaciones entre la actividad turística y la innovación tecnológica, las afectaciones de la pandemia al turismo, 
los destinos inteligentes y las iniciativas de desarrollo turístico de la ciudad de Portoviejo, entre otros trabajos que ayudaron a direccionar la revisión.

La búsqueda de información ha sido desarrollada a través de las principales bases de datos de revistas y repositorios digitales, tanto nacionales como internacionales a continuación se nombran algunas: Web of Science, Scopus, Science Direct, Scielo, Redalyc, Latindex, Google Académico o Dialnet, entre otros, en los cuales se han generado una serie de artículos publicados con revisión por pares anónimos (Rivera \& Félix, 2019); a lo que se le ha añadido las consultas en páginas web relacionadas a la temática turística gastronómica.

Para la clasificación de la información, se ha utilizado la metodología de pasos múltiples propuesta en Avraham $(2013,2016)$. Esta metodología propone la revisión minuciosa de literatura especializada, informes noticiosos de organismos oficiales y un mapeo de actores en el territorio, para recolectar información de primera mano, en este caso se ha utilizado la primera parte. Estas experiencias se han organizado en fichas estructuradas con formato homogéneo, para facilitar su análisis y comparación de contenidos.

\section{Resultados y Discusión}

\subsection{La innovación tecnológica}

Bajo una perspectiva generalizada de estudio, la innovación tecnológica (IT) aparece como un sistema complejo (Frenken, 2006). La innovación se define bajo cuatro clasificaciones: de producto, de proceso, de organización y de mercadotecnia; el primer tipo de innovación se refiere a cambios significativos en las características del producto o servicio, la segunda a nuevos métodos de producción y distribución, la tercera a la implementación de nuevos métodos organizativos y la cuarta a nuevos métodos de comercialización. (OECD, 2005, p. 23,24). La innovación no solo implica el que hacer sino también el cómo hacerlo para lograr la materialización desde las ideas a los negocios (Souto, 2015). En este sentido, se debe considerar que no todos los innovadores tienen una clara idea de la innovación en los diferentes sectores, por esta razón su implementación no ha tenido los resultados esperados (Vera, Alfonso, \& Reinoso, 2018).

El Manual de Oslo (OECD, 2005) recoge los criterios más generales para la medición de la innovación desde una perspectiva nacional, e introduce dos tipos de innovaciones: radical (basado en el cambio total o introducción de un método nuevo en cualquiera de las dimensiones expuestas) e incremental (consiste en mejorar los métodos en cualquiera de las dimensiones sin sustituir por completo la tecnología existente), determinado los indicadores de medición bajo la naturaleza de su impacto, gastos y difusión. Sin embargo, el crecimiento de la innovación se ha polarizado bajo la naturaleza del desarrollo de tecnologías.

En Latinoamérica la IT debe ser medida por sus capacidades tecnológicas; en el Manual de Bogotá, Jaramillo, Lugones, \& Salazar (2001) señalan que "existe un cuerpo de ideas de difusión relativamente amplia, que supone que las actividades de innovación tecnológica se concentran en los países desarrollados (PD) y su resultado es la creación de tecnologías incorporadas en la capacidad de producción” (p. 49). La velocidad con la que está evolucionando el proceso tecnológico hace que las innovaciones queden obsoletas en un corto periodo de tiempo (RICyT \& CAEU, 2009). Según BAI (agencia de innovación) (2010) "la globalización de los mercados y el desarrollo de la red digital han transformado de manera definitiva el valor que las empresas atribuyen a la información, hoy más estratégica que nunca, dada su necesidad de hacer propuestas coherentes, certeras y diferenciados". Lo que ha hecho imprescindible el apoyo en la IT para gestión de la competitividad de las empresas.

\subsection{La innovación tecnológica en los destinos turísticos inteligentes}

La innovación debe de ser promovida desde cada uno de los sectores, como lo señala el modelo de gestión de la 
innovación tecnológica para el desarrollo del turismo en un territorio, propuesto por Vera et al. (2018) (ver Figura 1), en el que se considera el resultado de la gestión turística desde una perspectiva inteligente. Este modelo recoge las cuatro dimensiones (producto, proceso, organización y mercadotecnia) propuestas en el Manual de Oslo (OECD/Eurostat, 2005) y contrasta su dinámica de gestión desde las esferas pública, privada y comunitaria, en función de la interacción con la demanda antes, durante y después de la visita.

Figura 1. Modelo estrella para la gestión de la IT.

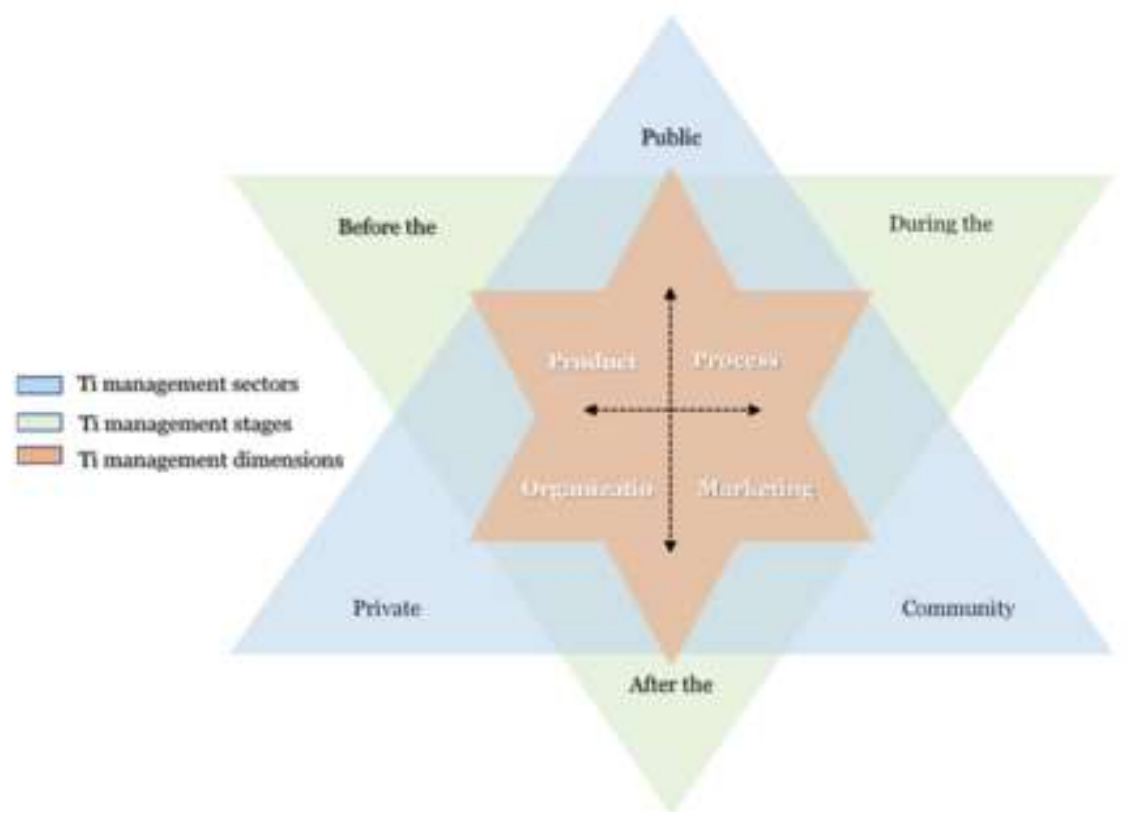

Fuente: Vera et al. (2018).

Aunque este modelo ofrece una perspectiva clara de los elementos que intervienen en la gestión del turismo con un enfoque de innovación, podrían presentarse gaps, en cuanto a su dinámica de interacción, o de elementos tecnológicos indispensables para su funcionamiento. Hjalager (2010) señala que el turismo ha sido una actividad que se ha caracterizado por su inmersión en el campo de la innovación. Desde esta óptica, Cosma, Paun, Bota, y Fleseriu (2014) agrega que la innovación tecnológica en el turismo debe ser analizada como un proceso permanente y dinámico.

Esto plantea la necesidad de analizar los territorios turísticos potenciales bajo la perspectiva de los DTI, para entender de mejor manera en que consiste la IT en el marco del turismo con un enfoque inteligente. En este contexto, un DTI, es un espacio innovador consolidado sobre la base del territorio y de una infraestructura tecnológica de vanguardia (López et al., 2015). Para Luque Gil, Zayas Fernández, y Caro Herrero (2015) los destinos turísticos inteligentes deben promover un desarrollo territorial inteligente. En este marco, Celdrán Bernabéu, Mazón López, y Giner Sánchez (2018) destaca la importancia del internet y la rápida evolución de las TIC para la gestión de la información en los DTI, y la planificación y gestión de ciudades y destinos inteligentes en el siglo XXI.

Los avances en conectividad, la irrupción de la web 2.0, el creciente uso de los dispositivos móviles y de las redes sociales ha propiciado un enorme impacto en el turismo, siendo uno de los sectores que más profundamente ha cambiado (SEGITTUR, 2015). Según la OMT (como se cita en Vera \& Rodney; 2018) las oportunidades de desarrollo sostenible, convergen con los avances tecnológicos. En este contexto, Vera y Rodney (2018) señalan que "en la actualidad lo más cercano a la construcción de un sistema de innovación tecnológica dentro de un destino turístico, se refleja parcialmente en las características tecnológicas de los destinos turísticos inteligentes (DTI)”. 


\subsection{La pandemia covid-19 y las afectaciones al turismo.}

La enfermedad por coronavirus iniciada en el 2019 (COVID-19) es un virus que se originó en China en la ciudad de Wuhan a finales del año 2019, evidenciado por la aparición de pacientes con infección respiratoria. Los casos fueron en aumento de forma acelerada en la ciudad epicentro del nuevo Coronavirus, extendiéndose por la provincia de Hubei y en algunas localidades del país asiático de China. Con el paso de los días, el virus comenzó a tener un alcance global, siendo declarado pandemia por la Organización Mundial de la Salud el 30 de enero de 2020 (Mahase, 2020).

El grado de connotación y descontrol de la enfermedad a nivel mundial se dio debido a que la mayoría de los países vieron en la aparición del nuevo Coronavirus una enfermedad que estaría posicionada solo en China, lo cual provocó que muchas naciones no tomen las correspondientes medidas para realizar controles sanitarios y de bioseguridad en sus puertos, aeropuertos y pasos de frontera, lo cual dio paso a que no solo haya miles de infectados y muertos en China sino en todo el mundo. El virus pasó de continente en continente, comenzando por Asia y Oceanía, pasando por Europa, luego América para finalmente reportarse casos en países de África.

Al 13 de junio de 2020, el coronavirus había infectado a más de 7,7 millones de personas de los cuales la cifra de muertes por este virus llegó a 429000 en todo el mundo. Estados Unidos es el país con mayor número de infectados y más de 115.000 fallecidos, seguido de Brasil con 850.000 contagiados y más de 42.000 muertes. En tercer lugar, se encuentra Rusia con más de medio millón de infectados. El resto de los países que conforman la lista con la mayor cantidad de contagiados se encuentran India, Reino Unido, España, Italia, Perú, Alemania, Irán, Turquía, Francia, Chile, México, Pakistán y Arabia Saudí (RTVE.es, 2020). La tasa de infectados y muertos varía de país en país dependiendo, del grado de restricciones y confinamiento a los cuales ha sometido a su población, la capacidad hospitalaria y servicios médicos, la toma temprano o tardía de medidas de cierre de pasos fronterizos y puertos, la cantidad de pruebas de COVID-19 a los cuales sometió a sus residentes, entre otros factores.

La pandemia ha traído cambios acelerados en diversos aspectos de las personas a nivel global, incluyendo los relacionados a las actividades económicas. Se ha evidenciado una precipitada entrada en vigor de cambios de costumbres y que, en las condiciones del aislamiento social y el confinamiento, ha motivado una aceleración inesperada. Uno de esos cambios ha sido el cambio de los procesos analógicos a los digitales, los cuales cambian el panorama de la realidad económico-social. El sistema financiero de varios países está siendo sometido a prueba tras la crisis financiera de 2007-2008, y como consecuencia de la paralización de la mayoría de las actividades económicas, existe una alta tasa de desempleo y una ola de empresas que empiezan a quebrar, evidenciándose en muchas de las personas y compañías deudas impagas que se comienzan a acumular por varios meses (Universidad de Belgrano, 2020). La incertidumbre en el sector económico y financiero en todos los países ha hecho que se disminuya el intercambio comercial, dadas las restricciones sanitarias en la carga y descarga de mercadería en los puertos de todo el mundo. Otro indicador de la grave crisis mundial se manifiesta por el decrecimiento de las economías de las principales naciones del orbe principalmente entre los países de América Latina. Debido al gran número de personas que han sufrido por la pérdida de sus empleos, los gobiernos se han visto en la necesidad de dar ayudas sociales lo cual ha provocado el recorte de gastos presupuestarios sufriendo las mayores consecuencias la inversión pública, para reorientarlas a la salud.

El turismo es uno de los sectores económicos más afectados por la pandemia del COVID-19, el cual ha obligado a aeropuertos a cerrar sus instalaciones, dejando a aviones en tierra, y por consecuencia derivando en cierre de hoteles y restricciones de viaje a prácticamente todos los países del mundo. La OMT detalla en su resumen ejecutivo del barómetro a mayo del 2020, una disminución de 67 millones de llegadas de turistas internacionales y derivando en pérdidas por alrededor de 80000 millones de USD. Así mismo, se estima que se encuentran entre 100 y 120 millones de puestos de trabajo en peligro (OMT, 2020). Las pérdidas de puestos de trabajo se originan en su mayoría en el cierre y declaraciones de quiebra de 
aerolíneas aéreas, agencias de viajes, operadoras y alojamientos turísticos los cuales se han visto perjudicados por el cierre de los aeropuertos y las restricciones de la movilidad.

Por regiones mundiales, Asia y el Pacífico, ha sido la primera región en sufrir el impacto de la pandemia, registrándose un descenso del 35\% en las llegadas durante el primer trimestre de 2020, seguida de Europa con un descenso del 19\%, seguida por las Américas con el 15\%, África con el 12\% y por último Oriente Medio con el 11\% de declive (OMT, 2020). El orden de la aparición del virus en las distintas regiones del mundo guarda similitud con los distintos porcentajes en cuanto a las caídas de llegadas de turistas, siendo que los primeros países en sufrir el efecto de la pandemia tuvieron que cerrar sus aeropuertos y puertos en primera instancia mientras en los países que no reportaban contagiados seguían las operaciones turísticas sin mayores complicaciones.

Ante ello, la OMT maneja diversos escenarios causados por la pandemia. Uno de ellos es, que la demanda interna se recuperará más rápido que la demanda internacional, debido a las restricciones de vuelo que existen a destinos fuera de las fronteras de cada país, cambios en las políticas para expedición de visas, la solicitud de documentos que comprueben no poseer COVID-19, entre otros. La demanda internacional se recuperaría en el cuarto trimestre y sobre todo en 2021 (OMT, 2020). Ante este panorama, muchos países han destinado sus pautas publicitarias al incentivo del turismo interno o de cercanía para que sus conciudadanos puedan visitar los atractivos turísticos de cada uno de sus países, así mismo se tuvieron que reenfocar los planes de desarrollo turístico para que puedan estar alineados a la nueva normalidad imperante producto de la pandemia.

Los escenarios de futuro dependen de la reapertura de las fronteras, tomando en cuenta que si las mismas se reabren a principios de julio la variación interanual concluirá con un declive del 58\% con respecto al 2019, si se lo realiza a principios de septiembre, el panorama finalizará con un declive del $70 \%$ y si se toma en cuenta un tercer escenario en el cual se reabren las fronteras en los primeros días de diciembre el descenso terminará en un 78\% (OMT, 2020). A pesar de lo descrito por la OMT, diferentes aerolíneas apoyadas por los entes gubernamentales han realizado vuelos de retorno para sus conciudadanos, permitiendo de esta forma dar un respiro en la economía de muchas de las compañías aéreas, así también, para el mes de junio se reportan en varios países los primeros vuelos domésticos debido a la desaceleración de la tasa de contagios.

Haciendo un resumen de impactos potenciales en 2020, se prevé que habrá pérdidas por entre 910.000 y 1,2 billones de USD en ingresos de exportación del turismo y finalmente entre 100 y 120 millones de empleos directos del turismo estarán amenazados (OMT, 2020). Los datos presentados obligan a los diferentes actores del turismo a generar propuestas, metodologías y soluciones que puedan ser aplicadas a corto, mediano y largo plazo para que millones de personas puedan seguir en la actividad y los destinos turísticos puedan recuperarse de las fuertes caídas acontecidas.

Como parte de las propuestas para la recuperación de la actividad turística, expertos, empresarios, académicos y personas relacionadas al sector turístico han planteado soluciones para que sean abordadas en miras de reactivar esta actividad económica, lo cual se refleja en el portal de la Red Interuniversitaria de Posgrados en Turismo, el cual muestra una recopilación de estudios y foros donde se discuten distintas aristas y soluciones para el turismo a nivel mundial (Red Interuniversitaria de Posgrados en Turismo, 2020)

\subsection{Los efectos económicos causados por el COVID-19 en Ecuador}

Ecuador es uno de los países más golpeados por la pandemia COVID-19 a nivel de América Latina (Bajaña Mendieta, 2020), reportándose hasta el 13 de abril de 2020 un total de 46356 casos confirmados y 3874 personas fallecidas (Ministerio de Salud Pública Ecuador, 2020). La provincia del Guayas es la provincia con mayor número de contagios con 14626 casos confirmados, seguido por la provincia de Pichincha con 5185 infectados y siendo la tercera provincia con mayor número de casos confirmados, Manabí, con 3248 personas contagiadas (Ministerio de Salud Pública Ecuador, 2020). A principios de abril, Ecuador fue el epicentro de la noticia mundial al darse a conocer el gran número de infectados sobre todo en la provincia 
del Guayas, teniendo su epicentro en la ciudad de Guayaquil, donde medios internacionales se dieron eco de la cantidad de personas que no podían recibir atención médica dada la saturación de los hospitales, así como de muchos más que morían en las calles y domicilios sin ser recogidos por las entidades forenses municipales o estatales.

La llegada de la pandemia al Ecuador ahondó aún más la grave crisis económica que desde octubre del 2019 vivía el país, encontrándose con un país endeudado y sin los suficientes recursos para enfrentar las secuelas de la pandemia, los cuales sumados al confinamiento obligatorio, el estancamiento de la producción y el insuficiente comercio electrónico, y más la caída del petróleo, tomó como consecuencia que una considerable cantidad de personas pierdan sus empleos, obligando al Estado ecuatoriano a asistir a la población que no tenía recursos para subsistir (Bajaña Mendieta, 2020). A pesar de los esfuerzos estatales para incentivar a las empresas para que no despidieran a sus trabajadores, muchos de los empresarios se sustentaron en la figura legal de "despido por fuerza mayor" o declarándose en quiebra. Hasta el 4 de mayo de 2020 se reportaron 508.000 personas que pidieron seguro de desempleo debido al cierre de empresas debido a que estas se encontraron, luego de varios días de bajo movimiento comercial, sin flujo de caja para pagar a sus empleados. Varias empresas han reducido del 25 al $50 \%$ el salario de sus trabajadores, siendo estas medidas un paliativo para no cerrar los servicios. En total, el sector productivo del Ecuador ha perdido cerca de 10000 millones de USD, siendo el detonante para que las empresas se declaren en quiebra y despidan personas (Voz de América, 2020).

Los efectos de la pandemia en la economía ecuatoriana también son sentidos por la actividad turística, los cuales, según datos presentados por la Ministra de Turismo Rosi Prado de Holguín, en el mes de marzo las pérdidas del sector superaron los 250 millones de USD, tomado por los datos de facturación real del Servicio de Rentas Internas (SRI). En contraste, Holbach Muñetón, presidente de la Federación Nacional de Cámaras de Turismo del Ecuador, estima que las pérdidas del sector turístico superan los USD 640 millones (El Comercio, 2020a). Se estima además que el 2020 tendrá una reducción de ingresos de alrededor del 70\% comparado con el 2019 en el sector turístico. Por otro lado, la Federación Nacional de Cámaras Provinciales del Ecuador (Fenacaptur) estima que la actividad turística en el Ecuador iniciará su recuperación a partir de marzo del 2021, cuando se empiecen a disipar los temores propios de la pandemia y exista un mayor deseo de viajar (El Universo, 2020).Por ahora, en la tercera semana de junio se están programando los primeros vuelos internos que ayudarán a reactivar el turismo interno, además de que las terminales terrestres del país se encuentran ofreciendo la apertura de rutas entre ciudades que poseen restricciones más flexibles dada la disminución de los casos reportados en cada uno de los cantones.

El panorama de los establecimientos de alojamiento y restauración en el Ecuador es crítico siendo el denominador común la reducción de trabajadores, existiendo el caso de hoteles que se quedaron con 80 de los 500 empleados que tenían antes de la pandemia. En otros casos, como en el de la restauración tuvieron que bajar a un alto personal en su nómina dado que los envíos a domicilio no compensaban las ventas que se tenían antes de la llegada del COVID-19 al Ecuador (El Universo, 2020). Una alternativa para reducir pérdidas económicas para algunos de los establecimientos de alojamiento ha sido la vinculación al programa propuesto por el MINTUR, permitiendo que los ecuatorianos que retornan del exterior pueden realizar el Aislamiento Preventivo Obligatorio (APO) en sus habitaciones (MINTUR, 2020b).

Los restaurantes y centros de diversión nocturna registran una caída del $63 \%$ en marzo y abril en comparación con igual período del 2019 (MINTUR, 2020b). Ante ello, los restaurantes han tomado alternativas para reducir las pérdidas económicas a través de la entrega de alimentos y bebidas a domicilio sea por medio de la comunicación telefónica, redes sociales o por medio de aplicaciones móviles. Así mismo, muchas empresas de alimentos se pudieron apalancar de empresas de "delivery" como Globo, Uber Eats, Rappi, entre otros. Por el contrario, los centros de diversión nocturna no han tenido la oportunidad de recuperarse económicamente debido a las restricciones de movilidad los cuales impedían a los ciudadanos concentrarse sobre todo en horas de la noche o en grupos de más de 10 personas.

Los profesionales en turismo han sufrido con mayor impacto los efectos de la pandemia en sus puestos laborales. Un 
estudio realizado por la Universidad Central del Ecuador junto a la Red de Profesionales de Turismo de Pichincha (Redprotur Pichincha, 2020), demuestra que el impacto sufrido es significativo dado que 7 de cada 10 profesionales encuestados manifestaron no tener un trabajo permanente en turismo, sea por suspensión de la actividad laboral o por terminación laboral o desempleo.

Ante la crisis provocada por el COVID-19 en Ecuador se han generado debates de opinión e investigación los cuales han sido llevados a las mesas de trabajo del MINTUR para la reactivación económica del sector. Las temáticas abordadas por cada una de las mesas fueron: cuantificación del impacto económico y laboral; ámbitos tributarios y crediticios; competitividad; promoción y cooperación; y desarrollo territorial. Dichos espacios de trabajo fueron conformados por técnicos del MINTUR y por representantes de los diferentes gremios (MINTUR, 2020c). Producto de labor realizada en las mesas especializadas se han elaborado protocolos de bioseguridad tanto para establecimientos de alimentos y bebidas (MINTUR, 2020d), como para los establecimientos de alojamiento turístico (MINTUR, 2020e). De igual forma, se ha elaborado una campaña publicitaria el cual motiva a los ecuatorianos a volver a visitar sus atractivos turísticos al terminar la emergencia sanitaria, buscando la reactivación del turismo interno y la dinamización de las economías locales. (MINTUR, 2020f).

De forma paralela, instituciones académicas han elaborado propuestas para la reactivación del sector turístico como lo expuesto en el documento 150 propuestas de acción para destinos, empresas y profesionales con miras hacia la regeneración del turismo en tiempos de crisis (Mathias et al., 2020), el cual propone directrices antes, durante y después de eventos que impacten gravemente la actividad. Entre las propuestas a tomar en cuenta post evento se da énfasis en la sostenibilidad, marketing, gobernanza y política pública, comunidad, consumidores, racionalización y oportunidades, y la elaboración o revisión de planes de gestión de crisis. Por otra parte (Félix, García, \& Vera, 2020) identifican una serie de códigos relacionados a estrategias orientadas a la recuperación de los ingresos por turismo, estas orientadas por una parte a las autoridades y por otra a los empresarios del sector.

\subsection{Portoviejo y su declaratoria de Ciudad Creativa Unesco (Gastronomía).}

El desarrollo del turismo gastronómico en Ecuador se refleja en la literatura científica mayoritariamente, a través de estudios de satisfacción de los turistas con el producto turístico, por lo que no se cuenta de suficiente información que resulte del análisis de la interrelación gastronomía- turismo (Vega et al., 2018). Para Sánchez \& López (2008) la gastronomía ecuatoriana se ha visto enriquecida por la pluriculturalidad, la diversidad de climas, así como la variedad de especias y productos que existen en el país. A pesar de esto existen muy pocos productos turísticos con valor agregado relacionados a la gastronomía, que sean capaces de promover por sí solos visitas organizadas al territorio. El potencial gastronómico radica en el turista interno que fluctúa entre varias regiones del país en sus periodos de descanso, la diversidad climática y la poca extensión territorial del país juegan un rol preponderante para la existencia de una variedad gastronómica poco aprovechada. Gracias a esto a lo largo del país encontramos zonas gastronómicas muy reconocidas, en el presente trabajo se las denomina corredores gastronómicos, debido a sus características físicas y entorno socio productivo. Concepto cada vez va tomando mayor preponderancia para la promoción de estos recursos gastronómicos a favor de la actividad turística.

En Manabí - Ecuador la actividad productiva se mantiene muy por debajo de los niveles básicos tanto para el sustento de sus familias, como para la generación de recursos adicionales, debido al poco valor agregado en la agricultura, ya que es una provincia netamente agrícola. En consecuencia, el sector de servicio de alimentos experimenta la misma carencia innovadora que los procesos de producción, comercialización y mercadeo, pese a contar con una buena imagen a nivel nacional, que la promueven como la mejor cocina del país (Cano Pita, 2013). Según Basurto, Párraga, \& Diéguez (2019) después de su estudio enfocado en la capital de la provincia de Manabí, al analizar la situación de las parroquias rurales, se pudo determinar que están en capacidad y tienen la potencialidad para formar parte de productos turísticos especializados en 
gastronomía, aprovechando su condición de combinarse con los recursos naturales y paisajísticos.

La gastronomía de Portoviejo hizo que esta ciudad y capital manabita entrara en la lista de Ciudades Creativas de la Organización de las Naciones Unidas para la Educación, la Ciencia y la Cultura (El Universo, 2019). La Red de Ciudades Creativas de la UNESCO se creó en 2004 para promover la cooperación hacia y entre las ciudades que identifiquen la creatividad como factor estratégico de desarrollo urbano sostenible (UNESCO, 2004). Esta importante declaratoria está relacionada con el gran potencial desarrollado alrededor de su recurso gastronómico. Para consolidar su desarrollo turístico, se lleva a cabo la iniciativa de fortalecer el destino turístico mediante el uso de la tecnología e innovación, que mejor manera que basado en un modelo de gestión turística inteligente, Portoviejo Ciudad Creativa UNESCO y Destino Turístico Inteligente (DTI).

\section{Conclusión}

La revisión de la literatura científica demostró que existe una perspectiva muy generalizada y compleja sobre la IT que implica no solo la acción del hacer, sino también el cómo hacerlo (know-how). Sin embargo, existe un concepto ampliamente difundido en el campo de la investigación de la IT, que la establece como un elemento esencial en la gestión de la competitividad de las empresas.

La IT en el turismo, se define como un proceso permanente y dinámico que debe ser gestionado desde cada uno de los sectores (público, privado y comunitario) que intervienen sobre un territorio, tomando como áreas de medición el producto turístico, el proceso de producción de los servicios turísticos, la organización y el marketing, los cuales deben ser evaluados en tres etapas básicas: antes, durante y después de la visita como lo propone Vera, et al. (2018).

La pandemia del COVID-19 ha golpeado severamente la economía mundial, y ha traído consigo cambios acelerados de los procesos, que inclusive han modificado hasta la forma en la que el ser humano se relaciona con su entorno social a efectos del confinamiento obligatorio, uno de esos cambios se observa en la transformación de procesos análogos a digitales, cambiando el panorama de la realidad económica y social de los países. En este sentido la OMT (2020) señala al turismo como uno de los más afectados por los estragos causados por la pandemia y pronostica el turismo interno como el que tendrá la mas pronta resiliencia. En Ecuador, el COVID-19, evidenció aún más la profunda crisis que vivía el país, sin los suficientes recursos para hacer frente a la pandemia que ocasionó el estancamiento de la producción, sumado a el insuficiente comercio electrónico y la caída del petróleo, y que en contexto turístico derivó en el cierre de establecimientos y la pérdida masiva de empleos.

Es necesario fortalecer las iniciativas turísticas desde el territorio, por ende la declaratoria de Portoviejo ciudad creativa UNESCO (gastronomía) conlleva un sin número de cambios para que pueda ser aprovechada de manera óptima por las autoridades locales. Los DTI ofrecen las herramientas adecuadas para una evolución del destino, sea cual fuere el concepto rector que se maneje, en este caso la gastronomía. El trabajo conjunto entre la academia y el gobierno local promueve la interacción entre los actores públicos y privados, iniciando mesas de diálogos que contribuyan a la creación de estructuras sólidas que faciliten la obtención de datos y toma de decisiones consensuadas, que redunden en el desarrollo empresarial del destino, creación de nuevos productos turísticos, acceso a los datos y nuevos flujos de visitantes.

Es necesario profundizar en la obtención de resultados a partir de las teorías consultadas, los destinos turísticos inteligentes, son una estrategia de desarrollo, no solo para la actividad turística, sino también, para la sociedad local en donde se desenvuelve. Existen varias metodologías y una larga lista de indicadores que deben ser evaluados en campo para obtener un diagnóstico inicial del territorio, en este caso la Ciudad Creativa de Portoviejo, estos indicadores están enmarcados en los cinco pilares base de los DTI como lo son la gobernanza, tecnología, accesibilidad, innovación y sostenibilidad. A partir de aquí se deberán proponer estrategias interinstitucionales de implementación en el territorio que permitan ir evaluando en el 
tiempo la evolución de dichos indicadores y por ende del DTI.

\section{Referencias}

Avraham, E. (2013). Crisis Communication, Image Restoration, and Battling Stereotypes of Terror and Wars : Media Strategies for Attracting Tourism to Middle Eastern Countries. 1-18. https://doi.org/10.1177/0002764213487733

Avraham, E. (2016). Destination marketing and image repair during tourism crises: The case of Egypt. Journal of Hospitality and Tourism Management, 28, 41-48. https://doi.org/10.1016/j.jhtm.2016.04.004

Berne, C., Garcia-Gonzalez, M., \& Mugica, J. (2012). How ICT shifts the power balance of tourism distribution channels. Tourism Management, 33(1), 205214. https://doi.org/10.1016/j.tourman.2011.02.004

Buhalis, D., \& Amaranggana, A. (2014). Smart Tourism Destinations. In Z. X. and I. T. (eds.) (Ed.), Information and Communication Technologies in Tourism (pp. 553-564). https://doi.org/10.1007/978-3-319-03973-2_40

El Comercio. (2020a). Demanda de servicio a domicilio por 'apps' se disparó y pedidos demoran. EL COMERCIO. https://www.elcomercio.com/actualidad/apps-delivery-demanda-demora-pedidos.html

El Comercio. (2020b). Pérdidas por covid-19 en Ecuador llegaron a USD 14 101 millones en mayo. EL COMERCIO. https://www.elcomercio.com/actualidad/perdidas-covid19-ecuador-economia-negocios.html

El Universo. (2019). Portoviejo entra en las Ciudades Creativas de la Unesco por su gastronomía. EL UNIVERSO. https://www.eluniverso.com/noticias/2019/10/30/nota/7582796/portoviejo-entra-ciudades-creativas-unesco-su-gastronomia

El Universo. (2020). Coronavirus frenó al turismo cuyos ingresos caerán un 70 \% en Ecuador durante el $2020 . \quad$ EL UNIVERSO. https://www.eluniverso.com/noticias/2020/05/10/nota/7836018/turismo-galapagos-ecuador-covid-19/

Félix, A., García, N., \& Vera, R. (2020). Participatory diagnosis of the tourism sector in managing the crisis caused by the pandemic (COVID-19). RIAT, $16(1)$.

Fernández, A., López, J., Moreno, L., Perles, J., Ramón, A., \& Such, M. (2017). Innovación y Destinos Inteligentes: Oportunidad para el Know How turístico español. Información Comercial Española ICE: Revista de economía, (894), 137-150. http://www.revistasice.com/index.php/ICE/article/view/1908/1908

Frenken, K. (2006). Technological innovation and complexity theory. Economics of Innovation and New Technology, 15(2), 137-155. https://doi.org/10.1080/10438590500141453

Gobierno Autónomo Descentralizado de Portoviejo. (2019). Agenda Portoviejo Ciudad Digital 2015-2019. Dirección de Informática, https://www.telecomunicaciones.gob.ec/wp-content/uploads/2019/08/AD-Portoviejo.pdf

Hjalager, A. M. (2010). A review of innovation research in tourism. Tourism Management. https://doi.org/10.1016/j.tourman.2009.08.012

HOSTELTUR. (28 de marzo de 2019). El mercado online en Europa en 5 cifras. https://www.hosteltur.com/127726_el-mercado-online-en-europa-en-5cifras.html

Huete, R. (2019). La digitalización de la economía y el fin del turismo en A. Álvarez-Sousa; A. Mantecón e I. Puertas-Cañaveral (eds.) Sociología del turismo. Pp. 299-321. Universidad de Alicante. http://rua.ua.es/dspace/handle/10045/103809

Iglesias-Sánchez, P., Correia, M., Jambrino-Maldonado, C. \& De Luque-Rojas, S. (2017). Retos en la gestión de destinos turísticos a partir de la innovación abierta. Estudios y perspectivas en turismo. 26(3):531-48. https://www.researchgate.net/publication/320108080_Retos_en_la_gestion_de_destino s_turisticos_a_partir_de_la_innovacion_abierta

Jaramillo, H., Lugones, G., \& Salazar, M. (2001). Normalización de Indicadores de Innovación Tecnológica en América Latina y el Caribe MANUAL DE BOGOTÁ. Red Iberoamericana de Indicadores de Ciencia y Tecnología/Organización de Estados Americanos, https://www.ovtt.org/wpcontent/uploads/2020/05/Manual_Bogota.pdf

Kim, D., \& Kim, S. (2017). The role of mobile technology in tourism: Patents, articles, news, and mobile tour app reviews. Sustainability (Switzerland), 9(2082), 1-45. https://doi.org/10.3390/su9112082

La Hora. (2019). Ecuador está 20 años atrás en innovación científica. LA HORA. https://www.lahora.com.ec/noticia/1102246384/ecuador\%C2\%A0esta-20anos-atras-en-innovacion-cientifica

La Sexta. (2020). España, el país con más reservas turísticas de todo el mundo para este verano. Atresmedia. https://www.lasexta.com/noticias/economia/espana-pais-mas-reservas-turisticas-todo-mundo-este-verano_202006095edf7264cbac95000136f4be.html

Linares, M., et al. (2020). Estimando el número de casos de COVID-19 a tiempo real utilizando un formulario web a través de las redes sociales: Proyecto COVID-19-TRENDS. SEMERGEN. (46), 114-116. https://www.sciencedirect.com/science/article/abs/pii/S1138359320301283?via\%3Dihub

López, A., Lancis, E., García, S., Alcantud, A., García, B., \& Muñoz, N. (2015). Informe destinos turísticos inteligentes: Construyendo el futuro. Segittur. https://doi.org/10.1007/s13398-014-0173-7.2

Luque Gil, A. M., Zayas Fernández, B., \& Caro Herrero, J. L. (2015). Los Destinos Turísticos Inteligentes en el marco de la Inteligencia Territorial: conflictos y oportunidades. Revista Investigaciones Turísticas. https://doi.org/10.14198/inturi2015.10.01 
Mahase, E. (2020). Covid-19: WHO declares pandemic because of "alarming levels" of spread, severity, and inaction. The BMJ. https://doi.org/10.1136/bmj.m986

Pécot, M., Veintimilla, C., \& Ricaurte, C. (2020). 150 propuestas de acción para destinos, empresas y profesionales con miras hacia la regeneración del turismo en tiempos de crisis. ESPOL, http://www.fcsh.espol.edu.ec/sites/fcsh.espol.edu.ec/files/150\%20propuestas\%20de\%20acci\%C3\%B3n\%20(1).pdf

Menchero Sánchez, M. (2020). Flujos turísticos, geopolítica y COVID-19: cuando los turistas internacionales son vectores de transmisión. Geopolítica(s). Revista de estudios sobre espacio y poder. 11 (Especial):105-14. https://revistas.ucm.es/index.php/GEOP/article/view/69249

Mendoza Mendizábal, A. C. (2016). Quito Como Destino Turístico Inteligente, Realidades De Una Ciudad En Crecimiento. [Tesis de Grado, Universidad de las Américas]. http://dspace.udla.edu.ec/handle/33000/6415

Ministerio de Salud Pública Ecuador. (2020). El MSP informa: Situación Coronavirus Covid-19 (13-06-2020) - Ministerio de Salud Pública. https://www.salud.gob.ec/el-ministerio-de-salud-publica-del-ecuador-msp-informa-situacion-coronavirus/

Ministerio de Turismo. (2020a). Turismo rural será clave para la reactivación del sector post Covid-19. Gestión Institucional. Recuperado (https://www.turismo.gob.ec/turismo-rural-sera-clave-para-la-reactivacion-del-sector-post-covid-19/).

Ministerio de Turismo del Ecuador. (2020b). COMUNICADO SOBRE ALOJAMIENTO - Ministerio de Turismo. https://www.turismo.gob.ec/comunicado-6/

Ministerio de Turismo del Ecuador. (2020c). Establecimientos de alojamiento turístico ya cuentan con un protocolo de bioseguridad - Ministerio de Turismo. https://www.turismo.gob.ec/establecimientos-de-alojamiento-turistico-ya-cuentan-con-un-protocolo-de-bioseguridad/

Ministerio de Turismo del Ecuador. (2020d). Ministerio de Turismo entregó protocolos de bioseguridad para establecimientos de alimentos y bebidas Ministerio de Turismo. https://www.turismo.gob.ec/ministerio-de-turismo-entrego-protocolos-de-bioseguridad-para-establecimientos-de-alimentos-y-bebidas/

Ministerio de Turismo del Ecuador. (2020e). Ministerio de Turismo presentó su campaña "Te Prometo Ecuador" - Ministerio de Turismo. https://www.turismo.gob.ec/ministerio-de-turismo-presento-su-campana-te-prometo-ecuador/

Ministerio de Turismo del Ecuador. (2020f). Ministra de Turismo: El trabajo conjunto nos permitirá reactivar al sector después de la emergencia por el COVID-19 - Ministerio de Turismo. https://www.turismo.gob.ec/ministra-de-turismo-el-trabajo-conjunto-nos-permitira-reactivar-al-sector-despues-de-laemergencia-por-el-covid-19/

Navarro Jurado, Enrique, y Germán Ortega (2020). Propuestas de reflexión desde el turismo frente al COVID-19 Incertidumbre, impacto y recuperación. Instituto Universitario de Investigación de Inteligencia e Innovación Turística de la Universidad de Málaga 18-23. https://www.i3t.uma.es/wpcontent/uploads/2020/03/Propuestas-Reflexiones-Turismo-ImpactoCOVID_i3tUMA.pdf

Navío-Marco, J., Ruiz-Gómez, L. M., \& Sevilla-Sevilla, C. (2018). Progress in information technology and tourism management: 30 years on and 20 years after the internet - Revisiting Buhalis \& Law's landmark study about eTourism. Tourism Management, 69, 460-470. https://doi.org/10.1016/j.tourman.2018.06.002

OECD/Eurostat. (2005). Manual de Oslo: Guía para la Recogida e Interpretación de Datos sobre Innovación. OCDE. https://doi.org/10.1787/9789264065659-es

OECD. (2005). Manual de Oslo: Directrices para la recogida e interpretación de información relativa a innovación. Oslo Manual (Vol. Third edit). http://scholar.google.com/scholar?hl=en\&btnG=Search\&q=intitle:Oslo+Manual:+Guidelines+for+collecting+and+interpreting+innovation+data\#0

Organización Mundial del Turismo. (2019). El turismo internacional sigue adelantando a la economía global. https://www.unwto.org/es/el-turismo-mundialconsolida-su-crecimiento-en-2019

Organización Mundial del Turismo. (2020). Barómetro OMT del Turismo Mundial mayo 2020 Con especial enfoque en el impacto de la COVID-19 (resumen). https://www.e-unwto.org/doi/pdf/10.18111/9789284421831

Ortiz, E., \& Noboa, P. (2020). Propuestas societarias y concursales para mitigar el impacto económico del covid-19 en Ecuador. X-Pendientes Económicos, 4(8), 38-48. https://ojs.supercias.gob.ec/index.php/X-pedientes_Economicos/article/view/110/39

Palomeque, F. L. (2016). Turismo y TIC. Notas sobre los procesos recientes de tecnificación de los Destinos Turísticos. Universidad de Alicante. Servicio de Publicaciones. 563-583. https://rua.ua.es/dspace/bitstream/10045/58774/1/Homenaje-Alfredo-Morales_26.pdf

Red Interuniversitaria de Posgrados en Turismo. (2020). REDINTUR - Recursos sobre turismo y COVID-19. https://red-intur.org/628-recursos-sobre-turismoy-covid-19.html

Redprotur Pichincha. (2020). Tercera nota técnica: Sondeo de la situación laboral profesionales en turismo. REDPROTUR Pichincha - Grupo de investigación Carrera de Turismo, Universidad Central del Ecuador, https://www.researchgate.net/profile/Enrique_Cabanilla2/publication/34 1525938_Sondeo_Profesionales_de_Turismo/links/5ec58d10458515626cbbdfd5/Sondeo-Profesionales-de-Turismo.pdf

RICyT, \& CAEU. (2009). Lisbon Manual Guidelines for the Interpretation of Available Statistical Data and the Construction of Indicators for Ibero-America's Transition to the Information Society. RYCIT, http://www.ricyt.org/wp-content/uploads/2019/09/manual_lisboaEN.pdf

Rivera, M., \& Félix, A. (2019). Planificación estratégica y gobernanza en la recuperación de destinos turísticos afectados por desastres socio-naturales. Un estado de la cuestión. Investigaciones Geográficas, (72), 235-254. https://doi.org/10.14198/INGEO2019.72.11

RTVE.es. (2020). El mapa mundial del coronavirus: más de 7,7 millones de casos y más de 429.000 muertos en todo el mundo - RTVE.es. https://www.rtve.es/noticias/20200613/mapa-mundial-del-coronavirus/1998143.shtml 
Research, Society and Development, v. 10, n. 2, e43210212754, 2021

(CC BY 4.0) | ISSN 2525-3409 | DOI: http://dx.doi.org/10.33448/rsd-v10i2.12754

Sánchez, S. \& López, T. (2008). La creación de productos turísticos utilizando rutas enológicas. Pasos Revista de turismo y patrimonio cultural, 6(2), 159171. http://www.pasosonline.org/Publicados/6208/PS0208_2.pdf

Santillán-Marroquín, W. (2020). El Teletrabajo en el COVID 19. CienciAmérica. 9(2), 1-6, http://cienciamerica.uti.edu.ec/openjo urnal/index.php/uti/article/view/289

SEGITTUR. (5 de junio de 2020a). La innovación, aliada del sector turístico. https://blog.segittur.es/la-innovacion-aliada-del-sector-turistico/

SEGITTUR. (2020b). Transparencia y otra información. https://www.segittur.es/es/conocenos/descripcion/index.html

Soares,A., Moreira, D., Parrerira, P., \& Shitsuka, R. (2018). Metodología da pesquisa científica. Universidade Federal de Santa Maria. https://repositorio.ufsm.br/bitstream/handle/1/15824/Lic_Computacao_Metodologia-Pesquisa-Cientifica.pdf?sequence=1

Soria, I. D., \& Joan, C. L. C. (2013). Reflexiones sobre el turismo de proximidad como una estrategia para el desarrollo local. Cuadernos de Turismo (32):6588, https://revistas.um.es/turismo/article/view/177421

Souto, J. E. (2015). Business model innovation and business concept innovation as the context of incremental innovation and radical innovation. Tourism Management, 51, 142-155. https://doi.org/10.1016/j.tourman.2015.05.017

UNESCO. (2004). Creative Cities Network. UNESCO. https://es.unesco.org/creative-cities/content/ciudades-creativas

Universidad de Belgrano. (2020). El impacto del COVID-19 en la economía global. Centros de Estudios de la nueva economía. http://repositorio.ub.edu.ar/bitstream/handle/123456789/8929/CENE_mayo_2020.pdf?sequence=1\&isAllowed=y

Vargas, Alfonso. (2020). Entender el turismo post-coronavirus: posibles escenarios. Asociación Española de Expertos Científicos en Turismo. https://aecit.org/uploads/public/DOCUMENTO.covid-19\%20y\%20turismo.pdf

Vega, V., Freire, D., Guananga, N., Real, E., Alarcón, M., \& Aguilera, P. (2018). Gastronomía ecuatoriana y turismo local. Revista Dilemas Contemporáneos: Educación, Política y Valores, 69(1), 1-17. https://doi.org/10.1017/CBO9781107415324.004

Vera, R., Alfonso, R., \& Reinoso, N. (2018). Design of an indicators system for the evaluation of technological innovation in the tourism destination of Puerto Lopez, Ecuador. Geojournal of Tourism and Geosites. https://doi.org/10.30892/gtg.25224-385

Vera, R., \& Rodney, A. (2018). La innovación tecnológica desde la perspectiva de los destinos turísticos: una revisión teórica. In VII Congreso Internacional La Universidad en el Siglo XXI. https://doi.org/10.1017/CBO9781107415324.004

Jacome, G. (2020). Empresas y trabajadores: entre los más impactados por el coronavirus en Ecuador. VOA. https://www.voanoticias.com/coronavirus/coronavirus-ecuador-desempleo

Wei-Han Tan, G., \& Keng-Boon, O. (2018). Gender and age: Do they really moderate mobile tourism shopping behavior? Telematics and Informatics, 35, 1617-1642. https://doi.org/10.1016/j.tele.2018.04.009

World Economic Forum. (2019). The Travel and Tourism Competitiveness Report 2019: Travel and Tourism at a Tipping Point. http://www3.weforum.org/docs/WEF_TTCR_2019.pdf 